PROGRESS REPORT AND FUTURE PLANS

FOR THE PROJECT TITLED

\title{
SOLID STATE, SURFACE AND CATALYTIC STUDIES OF OXIDES
}

GRANT NO. DE-FG-02-87ER13725

Submitted to the U.S. Department of Energy

For the period February 1, 1991 to December 31, 1991

File:911220

\author{
SOLID STATE, SURFACE AND CATALYTIC STUDIES OF OXIDES
}




\section{INTRODUCTION}

Selective oxidation of light alkanes is a potentially important route to convert the less valuable alkanes to more valuable chemicals such as unsaturated hydrocarbons and oxygenated compounds that are used in fuel mixtures and as feedstock. In specific operations, such processes can also be used to remove environmentally hostile chemicals while converting them into useful chemicals. For such processes to be commercially practical, high yields of the desired products must be obtained instead of the thermodynamically most favored products of carbon oxides. The objective of this research is to develop understandings regarding the interaction of molecules with catalytic surfaces and the relationship between the bulk and surface properties of oxides that would lead to the discovery of highly selective catalysts.

\section{OBJECTIVES FOR THIS PERIOD}

Work conducted in this period can be grouped into four projects, all of which deals with selective oxidation of alkanes and related reactions.

1) Investigation of the dependence of selectivity in the catalytic oxidation of $C_{2}$ to $C_{6}$ alkanes on the bonding characteristics of $\mathrm{VO}_{\mathrm{x}}$ units in vanadate catalysts.

2) Investigation of the effect of reducibility of the cations in the orthovanadate structure on the selectivity in the oxidative dehydrogenation of butane.

3) Investigation of the effect of $\mathrm{SiO}_{2}$ support and alkali metal ions on the oxidative dehydrogenation of butane.

4) Investigation of the effect of water in the oxidation of butan-2-one.

\section{PROGRESS AND FUTURE PLANS}

\section{Project 1: Relationship between selectivity in alkane oxidation and the bonding characteristics in vanadate catalysts.}

Over the past few years, we have been studying the oxidation of ethane, propane, 2 methylbutane, butane, and cyclohexane over a magnesium orthovanadate catalyst $\left(\mathrm{M}_{8_{3}}\left(\mathrm{VO}_{4}\right)_{2}\right)$. This catalyst is quite selective for oxidative deh ydrogenation of these alkanes except ethane. For the compounds that the catalyst is quite selective, the dependence of selectivity on the conversion of alkane shows similar behaviors: the selectivity for hydrogenation is high $(>70 \%)$ at very low conversions, but decreases as the conversion increases. Some typical data are shown in Table 1.

We discovered that the reaction data can be represented in a way that shows some unexpected and yet very interesting correlations. From the product distributions at different 
conversions of alkanes, one can calculate the number of oxygen molecules consumed by every molecule of alkane reacted. These calculated rates of oxygen consumption agree with the experimental values measured from the conversion of oxygen. When these rates of oxygen consumption were plotted against the rates of hydrocarbon consumption, a correlation was observed as that shown in Fig. 1. The slope of the points in this figure is the average number of oxygen molecules consumed by every hydrocarbon molecule reacted, that is, the stoichiometry for oxygen consumption. This number is about two. Thus the plot shows that for all the alkanes studied on $\mathrm{Mg}_{3}\left(\mathrm{VO}_{4}\right)_{2}$, which include ethane that reacts nonselectively to cyclohexane that reacts very selectively, the stoichiometries for oxygen consuniption take on very similar values.

Subsequently the reaction data for these alkanes on two other catalysts, $\mathrm{Mg}$ pyrovanadate $\left(\mathrm{Mg}_{2} \mathrm{~V}_{2} \mathrm{O}_{7}\right)$ and vanadyl pyrophosphate $\left((\mathrm{VO})_{2} \mathrm{P}_{2} \mathrm{O}_{7}\right)$ were also collected and plotted in the same manner. The results are shown in Fig. 2 and 3 , respectively, and some characteristic product distributions corresponding to the data in these figures are listed in Tables 2 and 3. Again it was found that in spite of the very different products and selectivities observed on the two catalysts, the stoichiometries for oxygen consumption take on rather narrow ranges of values. For $\mathrm{Mg}_{2} \mathrm{~V}_{2} \mathrm{O}_{7}$, these values are about two and four, and for $(\mathrm{VO})_{2} \mathrm{P}_{2} \mathrm{O}_{7}$, they are about one and four.

To our knowledge, this is the first time such observations were made. We believe that the small ranges of stoichiometries for oxygen consumption should be related to the bonding characteristic of oxygen ions in the catalysts and the manner in which these oxygen ions participate in the selectivity-determining steps. We are actively trying to generate an explanation for these data.

In the process of trying to understand these stoichiometric relationships, we noticed that there were no detailed kinetic analyses of selective oxidation reactions in which lattice oxygen is the proximate source of oxygen in the reaction. In particular, no kinetic models are available that mathematically (1) address the rate of diffusion of lattice oxygen in the observed kinetics; (2) describe the distribution of surface active sites that are in the oxidized form, the reduced form, and covered by surface intermediates; and (3) describe the deuterium isotope effect when the rate of reoxidation of the reduced catalyst is a slow step.

Efforts were spent to derive a kinetic model for a generalized selective oxidation reaction. Most aspects of the model have been recognized by previous workers except one, which was necessary to bring the mathematical formulation to a closure. We proposed an equation that related the total number of oxygen atoms consumed by a surface intermediate to the jump frequency of oxygen ions in the lattice between lattice points and the surface residence time of the intermediate. With this new equation, analytical expressions for rates, kinetic isotope effects, and surface concentrations of active sites that are oxidized, reduced, or covered with intermediates were obtained. The paper reporting this analysis has been accepted for publication.

In addition to analyzing the kinetic model for a generalized selective oxidation reastion, the application of the concept of the model to the selective epoxidation of ethylene to ethylene oxide on silver was also investigated. Thus the epoxidation reaction was also analyzed and the 
resulting rate expressions were used to explain the kinetic isotope effect observed experimentally. A publication on this work has been accepted.

Project 2: Effect of reducibility of cations in orthovanadates on the selectivity in butane oxidation.

We have been using as a guideline to our thinking that the way lattice oxygen is bonded in an oxide is an important factor determining selectivity in oxidative dehydrogenation of alkanes. In particular, the reducibility of the cations in the M-O-M bonds determines the ease of abstraction of the bridging oxygen, which in turn determines the rate of oxygen addition to a surface intermediate. In order to test the validity of this concept, we proceeded to study the catalytic properties of orthovanadates of $\mathrm{Nd}, \mathrm{Sm}, \mathrm{Cr}, \mathrm{Fe}, \mathrm{Mg}, \mathrm{Zn}, \mathrm{Ni}$, and $\mathrm{Cu}$. These cations were chosen based on their different aqueous reduction potentials. In the last progress report, we reported that steady state butane oxidation reactions had been performed on thess orthovanadates, and the selectivity for butenes and butadiene followed the order:

$\mathrm{Mg}>\mathrm{Nd}, \mathrm{Sm}>\mathrm{Zn}>\mathrm{Ni}>\mathrm{Fe}, \mathrm{Cr}>\mathrm{Cu}$

which paralleled their reduction potentials.

The concept that the reducibility of these cations affected the reactivity of the lattice oxygen bonded to it can be used to interpret the results only if the lattice oxygen indeed participated in the reaction, especially in the selectivity determining step. To confirm this, pulse reaction studies were conducted using butane pulses in the absence of gaseous oxygen on five orthovanadates: $\mathrm{Mg}, \mathrm{Zn}, \mathrm{Ni}, \mathrm{Fe}$, and $\mathrm{Cu}$. In all cases, the selectivities for dehydrogenation were very similar to those observed in the steady state reaction. Thus the assumption that lattice oxygen was important was justified.

In addition, the reducibility of selected orthovanadates was investigated using temperature programmed reduction. Except for Fe orthovanadate, the on-set temperature for reduction increased with increasing aqueous reduction potential of the cation, which suggested that these aqueous values are good approximation for the relative reduction potentials of the cations in solids of similar structures. We are preparing publications to report these results.

Project 3: The effect of $\mathrm{SiO}_{2}$ support and alkali metal ions on the oxidative dehydrogenation of butane.

In the last progress reported, it was stated that when the oxidative dehydrogenation of butane was studied over unsupported or supported vanadia on $\mathrm{SiO}_{2}$ at $500^{\circ} \mathrm{C}$, the selectivity for dehydrogenation decreased with the vanadia loading on $\mathrm{SiO}_{2}$ and approached the low selectivity of the unsupported sample at high loadings. There was a concern of whether this effect was due to impurities in the silica.

To investigate the effect of impurities, catalysts of different vanadia loadings were 
prepared on three different types of silica: Davison 952 that was briefly washed with dilute acid and still contained about $500 \mathrm{ppm} \mathrm{Na}$ and other impurities $(\mathrm{Cd}, \mathrm{Ti}, \mathrm{Al}$ and $\mathrm{Zr}$ ), Davison 952 thoroughly washed with acid and contained $<10 \mathrm{ppm} \mathrm{Na}$ but still some of the other impurities, and Cabosil that contained $<10 \mathrm{ppm} \mathrm{Na}$ and very little other impurities. Catalysts containing 1 and $10 \mathrm{wt} \%$ vanadia on the support were prepared and investigated for their catalytic properties.

The results of these experiments showed that the impurities in silica have some effects. They increased the selectivity for dehydrogenation, in particular enhancing the production of butadiene. However, there was a much larger effect due to the vanadia loading. Since different loadings resulted in different distributions of vanadia species of different bonding to silica, the results suggested that the nature of the bonding of vanadium and oxygen ions are the dominant factor in determining selectivity. We are planning to gain deeper insight into the structuralselectivity relationship by further characterization of the catalysts.

\section{Project 4: The effect of water in the oxidation of butan-2-one.}

In many selective oxidation reactions, water is added in the feed mixture. There are a number of proposed functions of water, which varied from acting as a heat transfer agent, to changing the acidity of the surface, to removal of surface carbonaceous residues. In this project, we intended to studying how does water affect activity and selectivity in the oxidation of butan2-one, which was chosen because it could provide information on the selectivity for carboncarbon bond cleavage versus carbon-oxygen bond formation. Vanadium oxide samples containing different amounts of potassium and cesium were used as catalysts.

In all experiments, acetic acid and acetaldehyde were the major products. Other important products included 2,3-butanedione and carbon oxides. The addition of water in the feed increased the activities of the catalysts as well as the selectivities to acetic acid, acetaldehyde and 2,3butanedione. The modification of vanadia with alkali metal ions increased the activity.

The change in activity due to water and alkali modification correlated well with the weight loss in the TGA measurements in the presence of oxygen and with the surface carbon to vanadium ratios as determined by XPS. Thus it was concluded that the main effect of water and alkali modification was to reduce the formation of carbonaceous deposits on the catalyst. 


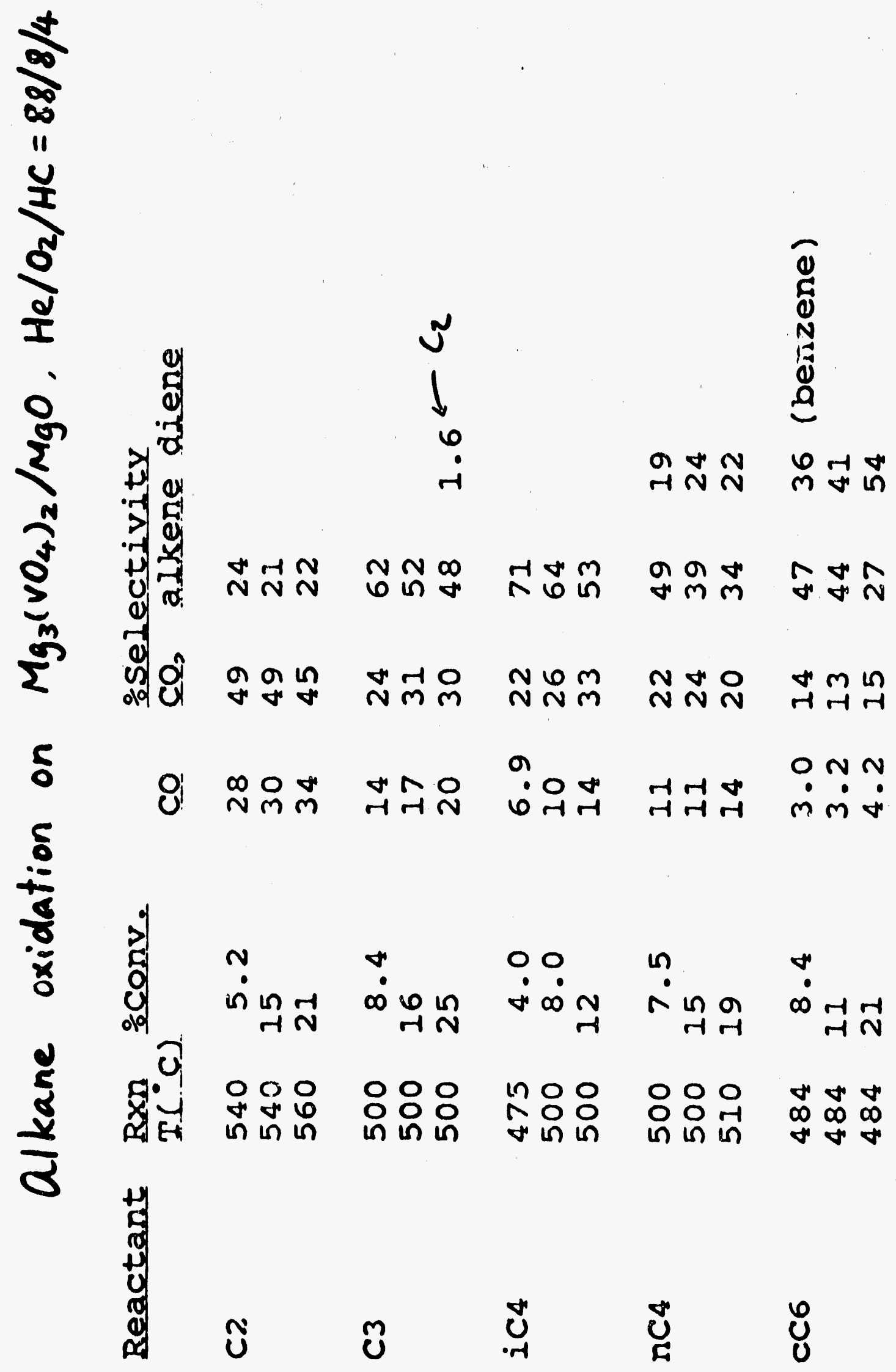



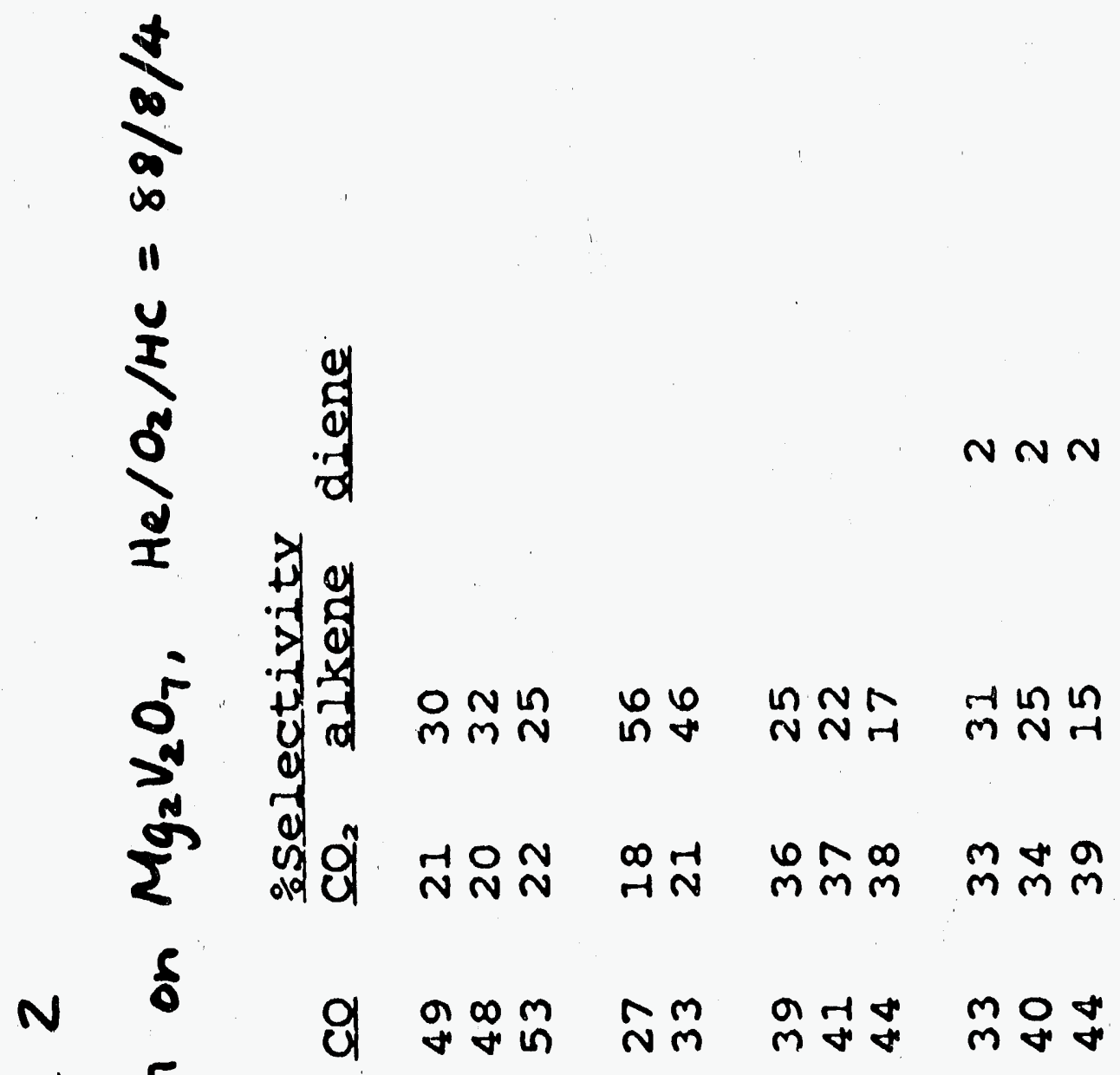

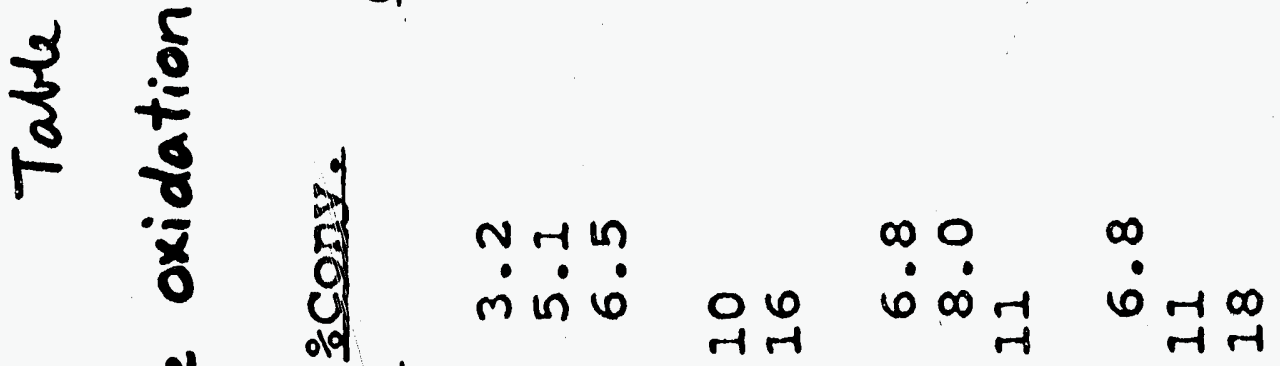

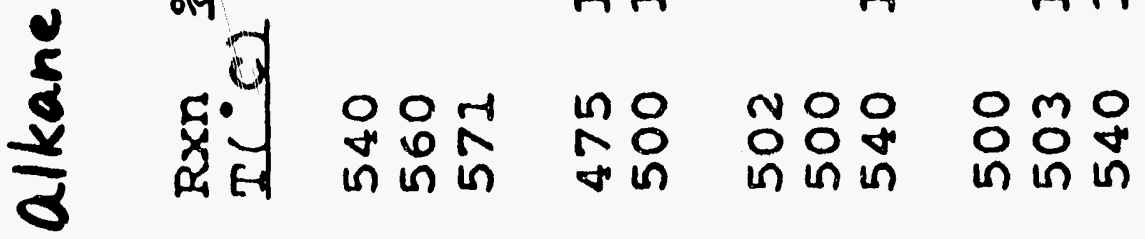

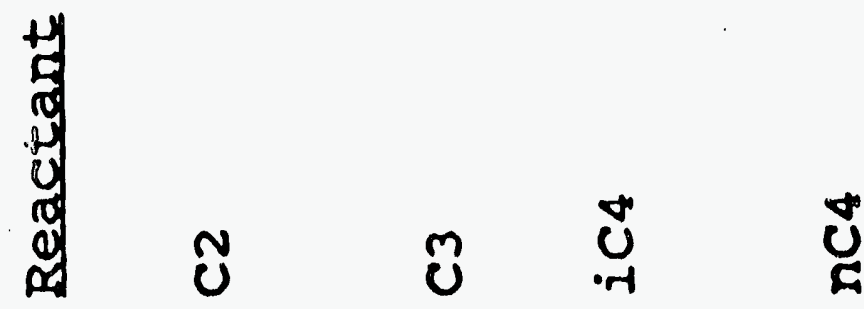




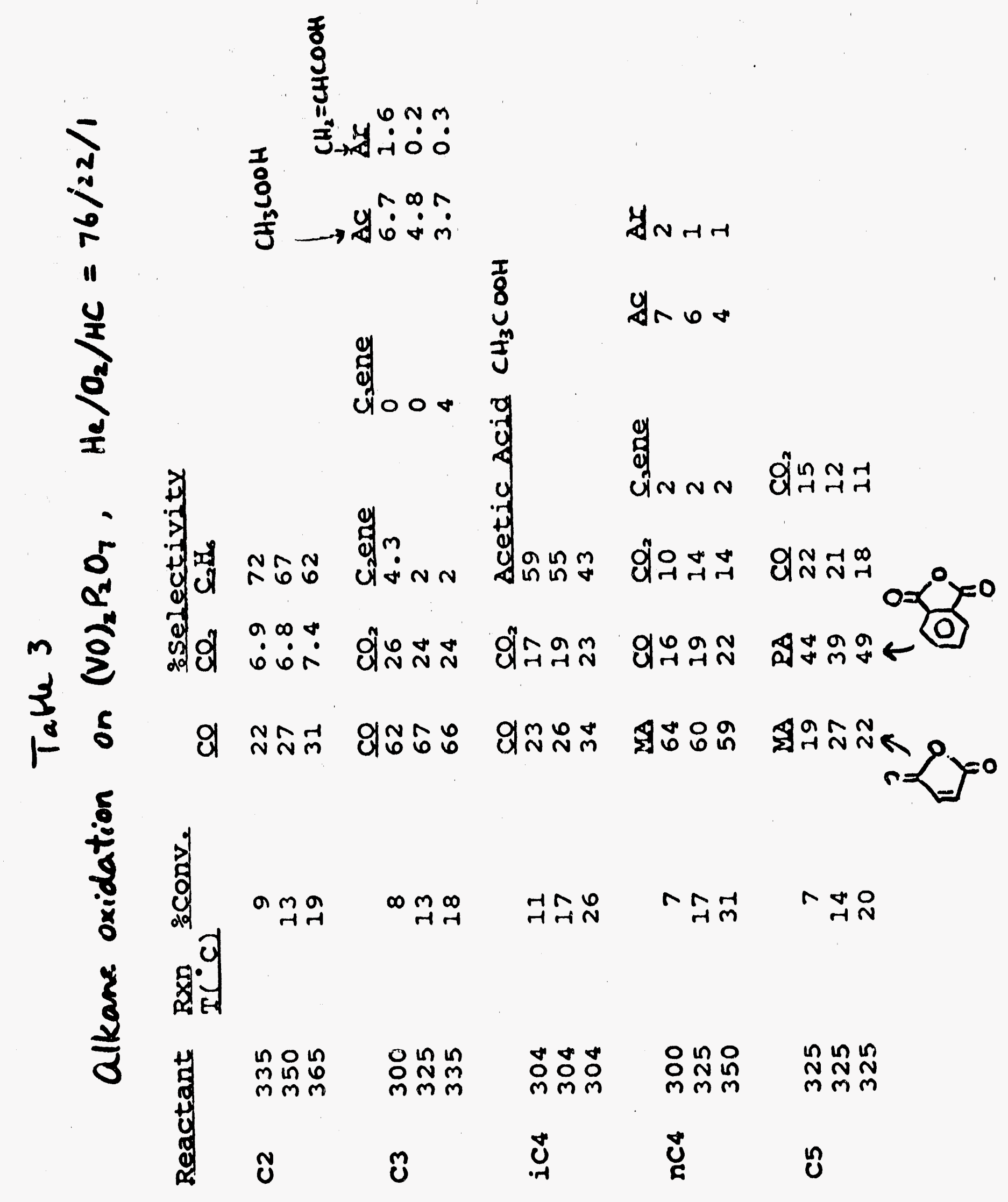


Figure I

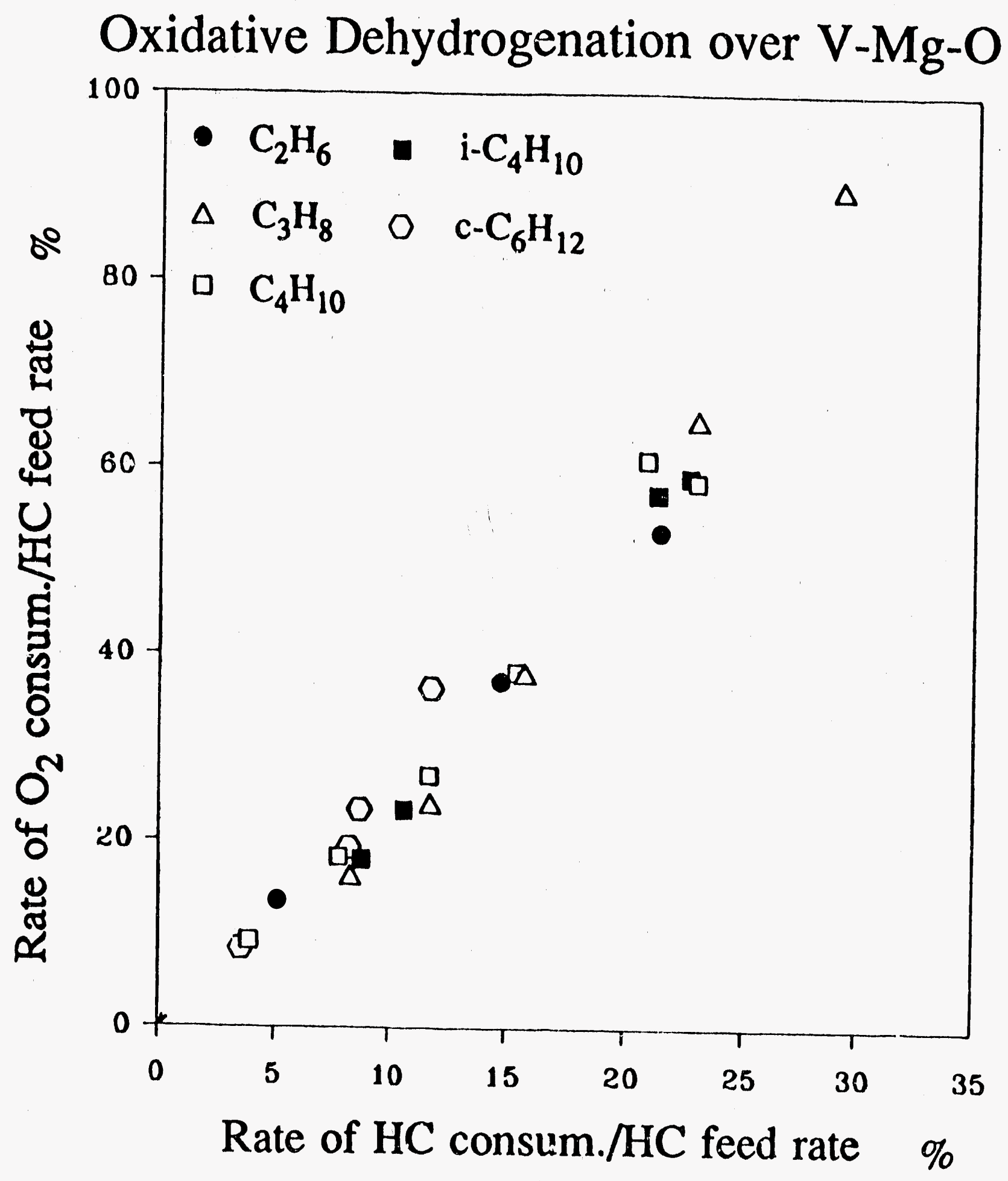


Figure 2

$$
\mathrm{Mg}_{2} \mathrm{~V}_{2} \mathrm{O}_{7}
$$

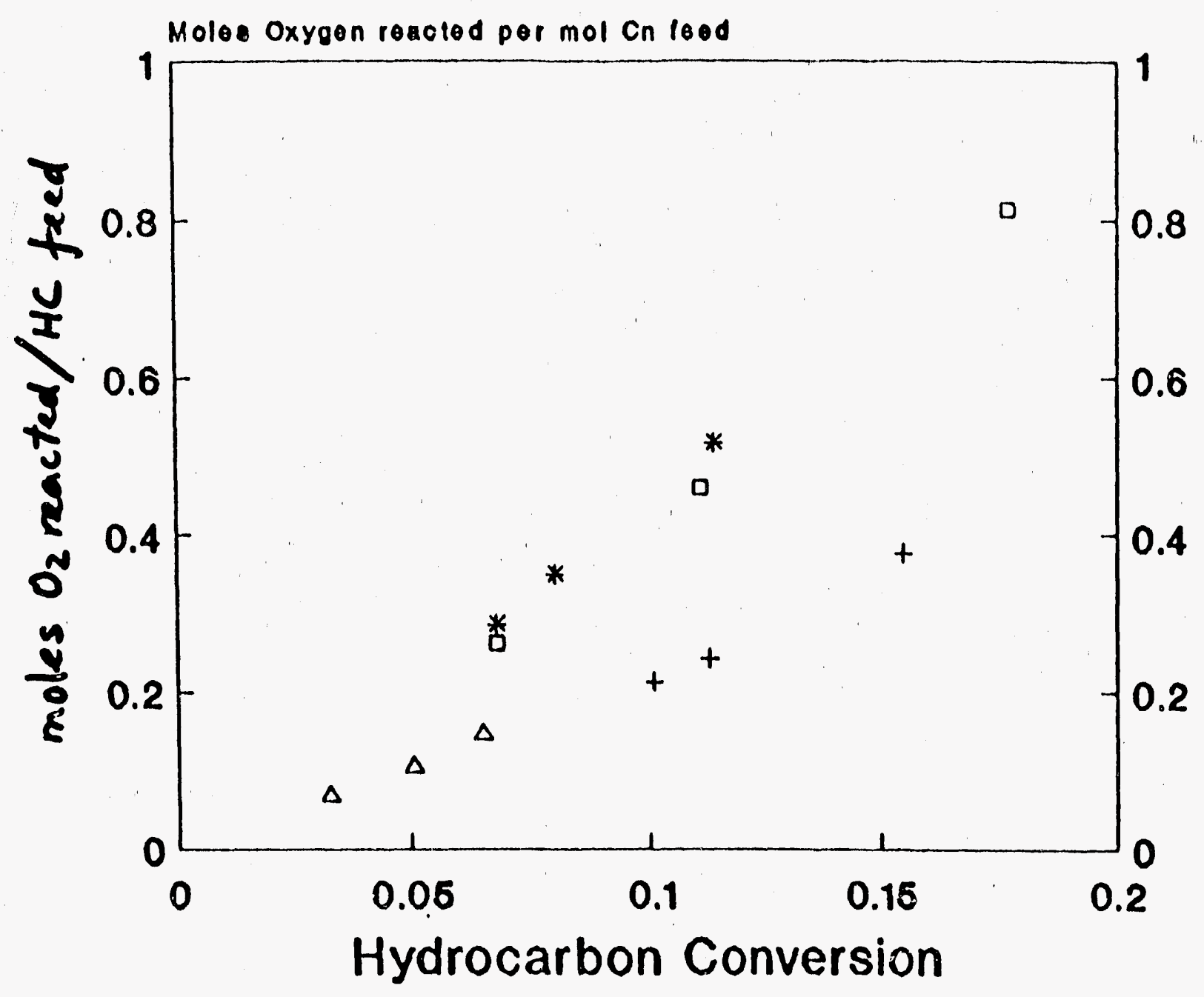

Reactant

$$
\begin{aligned}
& \Delta \quad \text { Ethano } \\
& * \quad \text { loo-Butano }
\end{aligned}
$$

$$
\begin{aligned}
& + \text { Propane } \\
& \text { - n-Butane }
\end{aligned}
$$


Figure 3

$$
(V O)_{2} P_{2} O_{7}
$$

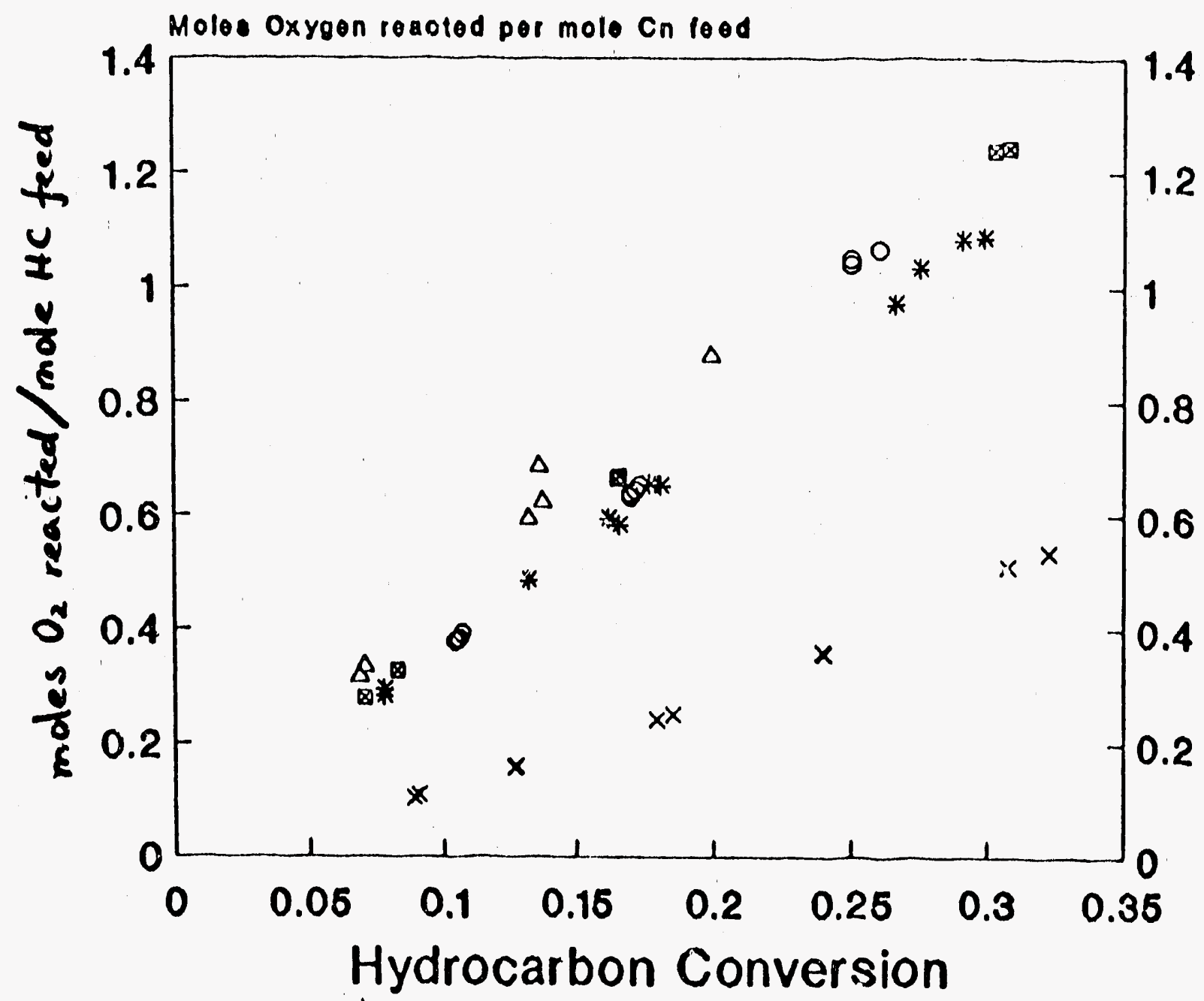

Reactant

- n-Butane

$x$ Ethane
- I-Butano

$\triangle$ Pontano
* Propano

Dentano

reprints and prepients removed ard cyeled separatily 

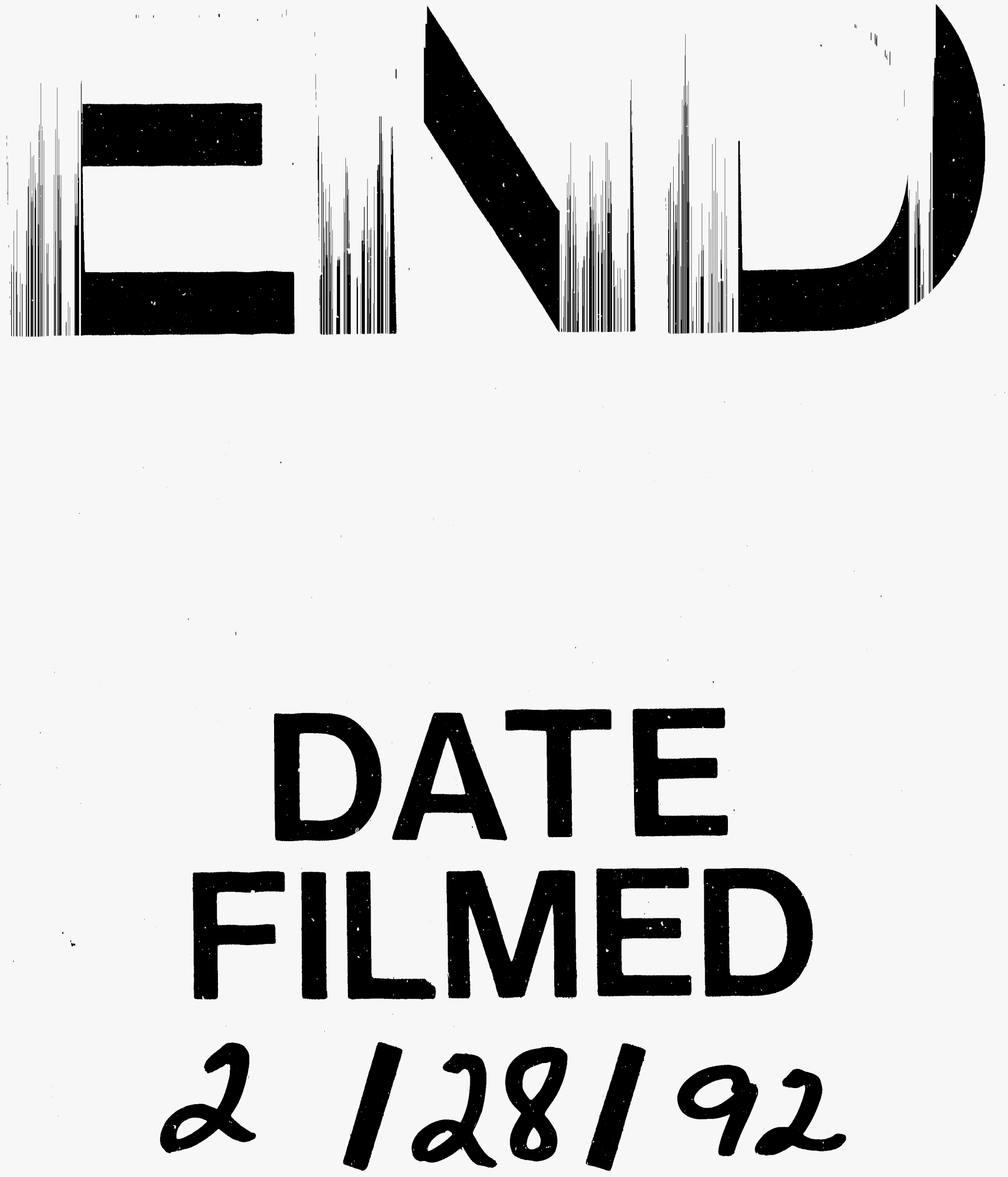